\title{
Review of e-GP
}

As a self-confessed Luddite, I will admit to having a few reservations before trying e-GP. In addition to the hassle of dealing with temperamental software, I often find online learning to be impersonal and lacking in the satisfaction to be had from reading a book. However, six modules later, e-GP ${ }^{1}$ has challenged many of my preconceptions.

E-GP is surprisingly easy to use even for those of us who think Wi-Fi and Wii Fit are interchangeable entities. The layout is clear and attractive with lots of pictures to hold the user's attention. In addition, the first module is a very helpful and easy to understand guide to using e-GP.

Pablo Picasso is quoted as having said, 'Computers are useless. They can only give you answers.' If used in conjunction with the online new personal evaluation program (nPEP) ${ }^{2}$ then e-GP is set to prove him wrong. To my knowledge, this is the only online learning resource that helps the user find out what they need to know as well as how to learn it.

As with many online resources there is capacity to create a 'learning path'. This is a great tool for focusing one's study. Equally, a record of learning is available, extremely helpful in the typically lastminute panic to collate evidence for appraisal. Unusually, a record of the time spent for each module is shown, making calculation of CPD hours less arduous. My new found, revalidation-led craving for certificates is also sated by the ability to print these for completed modules.

There is a handy facility to record any note-worthy thoughts during the module. I continue to prefer to scribble with pen and paper but can see the use for more computer-orientated colleagues.

As a GP-mum hybrid, with a toddler who has a talent for pressing 'off' buttons, the facility to stop the module mid-way through and then resume is ingenious.

So far I have completed a variety of modules from different subject areas: ankle pain, postnatal depression, equality legislation, allergies in children, principles of palliative care, and how to learn with eGP. These vary slightly in duration and format, but I can honestly say I learned something new from each of them.

Common to each module is an interactive format which I particularly enjoyed. Case scenarios are frequently used along with regular quizzes, which kept my mind on track. Despite this, online learning remains a solitary process and not quite a replacement for teacher-pupil interaction. However, with regular points for reflection and feedback, I felt these modules were a close second.

The modules vary in depth, with one of the modules I completed being quite superficial. However, guidance is given concerning further learning resources, negating this worry. Overall, given the range of users, which will include trainees and GPs, both old and new alike, I feel the knowledge level was well pitched. If used as the starting point for learning, I feel these modules have considerable potential.

To conclude, I feel this is an extremely useful tool for learning that is at least equal to its online contemporaries. It is an invaluable resource in the quest to achieve our CPD goals and, as such, I stand converted.

\section{Faye McCleery}

\section{REFERENCES}

1. Department of Health and NHS and RCGP. e-GP, eLearning for General Practice. http://e-

lfh.org.uk/projects/egp/index.html (accessed 6 Jul 2009).

2. Royal College of General Practitioners. RCGP Scotland Online Assessment Application for GPs. http://www.npep.org.uk//\# (accessed 9 Jul 2009).

DOI: 10.3399/bjgp09X453909 